\title{
The spectral periodicity of the spinon continuum in quantum spin ice
}

\author{
Gang Chen ${ }^{1,2,3 *}$ \\ ${ }^{1}$ State Key Laboratory of Surface Physics, Department of Physics, Fudan University, Shanghai, 200433, China \\ ${ }^{2}$ Center for Field Theory 83 Particle Physics, Fudan University, Shanghai, 200433, China and \\ ${ }^{3}$ Collaborative Innovation Center of Advanced Microstructures, Nanjing, 210093, China
}

(Dated: August 22, 2017)

\begin{abstract}
Motivated by the rapid experimental progress of quantum spin ice materials, we study the dynamical properties of pyrochlore spin ice in the $U(1)$ spin liquid phases. In particular, we focus on the spinon excitations that appear at high energies and show up as an excitation continuum in the dynamic spin structure factor. The keen connection between the crystal symmetry fractionalization of the spinons and the spectral periodicity of the spinon continuum is emphasized and explicitly demonstrated. When the spinon experiences a background $\pi$ flux and the spinon continuum exhibits an enhanced spectral periodicity with a folded Brillouin zone, this spectral property can then be used to detect the spin quantum number fractionalization and $\mathrm{U}(1)$ spin liquid. Our prediction can be immediately examined by inelastic neutron scattering experiments among quantum spin ice materials with Kramers' doublets. Further application to the non-Kramers' doublets is discussed.
\end{abstract}

\section{INTRODUCTION}

The three-dimensional (3D) U(1) quantum spin liquid (QSL) is an exotic quantum state of matter and is characterized by fractionalized spinon excitation and emergent $\mathrm{U}(1)$ gauge structure ${ }^{1}$. Since the spinons are gapped, the low-energy property of the state is described by a compact $\mathrm{U}(1)$ quantum electrodynamics in $3 \mathrm{D}^{1}$. This interesting state was proposed more than one decade ago $^{1-3}$. Recently, there has been a very active search of this exotic state among the rare-earth pyrochlore quantum spin ice $(\mathrm{QSI})^{4}$ materials $^{5-41}$. Despite the abundance of the QSI materials and possible experimental evidences, the identification of U(1) QSL has not been achieved in any candidate material.

To confirm the U(1) QSL, one needs to identify the emergent gauge structure and/or the fractionalized spinon excitation. From the theoretical perspective, these two things are related since the fractionalized excitation naturally emerges in the deconfined phase of the lattice gauge theory. Thus, identifying the emergent gauge structure and finding the fractionalized spinon excitations are equivalent. For the realistic pyrochlore QSIs, the gauge photon and the spinon have drastically different energy scales ${ }^{1,8,9}$. The gauge photon is the very low energy excitation that operates on the spin ice manifold $^{28,36}$, while the spinons are the much higher energy excitations that violate the spin ice rule ${ }^{1}$. Practically speaking, the large energy-scale difference between the gauge photon and spinons suggests that the spinon excitation might be a better experimental direction to search for. Therefore, we focus on the experimental signature of the spinon excitation and explore the spectral structure of the spinon continuum in the U(1) QSL in this paper. In particular, we point out that the emergent background $\mathrm{U}(1)$ gauge flux of the ground state enriches the $\mathrm{U}(1)$ QSLs by creating distinct translational symmetry fractionalization for the spinons. In the case that the spinon experiences a $\pi$ background flux, there is an enhanced spectral periodicity with a folded Brillouin zone in the spinon continuum that can be revealed by the dynamic spin structure factor in an inelastic neutron scattering (INS) measurement. The enhanced spectral periodicity is certainly not a property of a conventional paramagnet and thus represents an unique experimental signature of the U(1) QSL with the $\pi$ flux.

The enhanced spectral periodicity of the spinon continuum in the U(1) QSL with $\pi$ flux, that we discover here, is very analogous to the fractional charge excitation in the fractional quantum Hall states ${ }^{43,44}$. Over there, the global U(1) charge conservation gives the fractional charge quantum number to the fractionalized excitation. In our case, it is the translation symmetry that is fractionalized and renders the enhanced spectral periodicity to the spin continuum. Both of these results are examples of symmetry enriched topological orders, where the

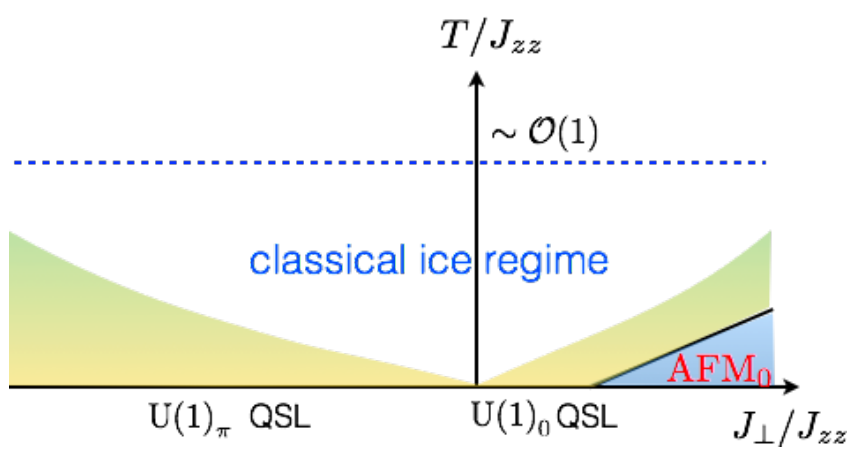

FIG. 1. (Color online.) The schematic phase diagram of the XXZ model on the pyrochlore lattice. The $\mathrm{AFM}_{0}$ stands for the magnetic ordered state that is proximate to the $\mathrm{U}(1)_{0}$ $\mathrm{QSL}^{42}$. The colored region refers to the QSI regime in which the quantum fluctuation gradually releases the classical spin ice entropy. The QSLs appear as the ground states at zero temperature, while the $\mathrm{AFM}_{0}$ extends to finite temperatures. The solid lines indicate a finite temperature magnetic ordering transition. The dashed line indicates the crossover temperature from the high temperature paramagnetic regime to the spin ice regime. See the main text and Tab. I for details. 


\begin{tabular}{lll}
\hline \hline $\mathrm{U}(1)$ QSLs & $\mathrm{U}(1)_{0}$ QSL & $\mathrm{U}(1)_{\pi}$ QSL \\
\hline Exchange Coupling & $J_{\perp}>0$ & $J_{\perp}<0$ \\
Background U(1) Flux & 0 Flux & $\pi$ Flux \\
Heat Capacity & $C_{v} \sim T^{3}$ & $C_{v} \sim T^{3}$ \\
Proximate XY Order & Keep Translation & Enlarged Cell \\
Spectral Periodicity & Not Enhanced & Enhanced \\
\hline \hline
\end{tabular}

TABLE I. Physical properties of the $\mathrm{U}(1)_{0}$ and $\mathrm{U}(1)_{\pi}$ QSLs.

symmetry not only makes the topological ordered phases finer ${ }^{45-48}$ but also makes the topological order more visible from the experimental point of view.

The following part of the paper is organized as follows. In Sec. II, we introduce the XXZ model as the parent model to extract the $\pi$-flux U(1) QSL in the frustrated and perturbative regime. In Sec. III, we explain the translational symmetry fractionalization and predict its consequence on the spectral periodicity of the spinon continuum. In Sec. IV, we explictly compute the spinon continuum with the parton-gauge contruction for the XXZ model. In Sec. V, we discuss the candidate materials and the related experimental consequences.

\section{MODEL HAMILTONIAN AND PERTURBATIVE ANALYSIS}

We start with the spin- $1 / 2$ XXZ model on the pyrochlore lattice. This model is the parent model for the pyrochlore $\mathrm{QSI}^{1}$. The realistic spin models for pyrochlore QSI contain more interactions ${ }^{8,9,12,14}$, but the simple XXZ model already realizes and captures the generic property of the pyrochlore ice U(1) QSL in the perturbative Ising regime. Therefore, we deliver our theory through the XXZ model but emphasize the modelindependent universal and generic properties of the $\mathrm{U}(1)$ QSL. This model is defined as

$$
\mathcal{H}_{\mathrm{XXZ}}=\sum_{\langle i j\rangle} J_{z z} S_{i}^{z} S_{j}^{z}-J_{\perp}\left(S_{i}^{+} S_{j}^{-}+S_{i}^{-} S_{j}^{+}\right),
$$

where $J_{z z}>0$. The phase diagram of the specific XXZ model is given in Fig. 1 and explained in the remaining part of the paper. In the regime with $\left|J_{\perp}\right| \ll J_{z z}$, the third-order degenerate perturbation theory yields an effective Hamiltonian that acts on the extensively degenerate spin ice manifold. The effective model is a ring exchange model with ${ }^{1}$

$$
\mathcal{H}_{\text {eff }}=-\frac{12 J_{\perp}^{3}}{J_{z z}^{2}} \sum_{\bigcirc_{\mathrm{p}}}\left(S_{i}^{+} S_{j}^{-} S_{k}^{+} S_{l}^{-} S_{m}^{+} S_{n}^{-}+h . c .\right),
$$

where " $i, j, k, l, m, n$ " are the six vertices on the elementary hexagon (" $\square_{p}$ ") of the pyrochlore lattice. To reveal the U(1) gauge structure, one introduces the lattice gauge fields as $E_{\boldsymbol{r} \boldsymbol{r}^{\prime}} \simeq S_{\boldsymbol{r} \boldsymbol{r}^{\prime}}^{z}, e^{i A_{\boldsymbol{r} \boldsymbol{r}^{\prime}}} \simeq S_{\boldsymbol{r} \boldsymbol{r}^{\prime}}^{ \pm}$, where $\boldsymbol{r}, \boldsymbol{r}^{\prime}$ label the centers of the tetrahedra and form a diamond lattice. The effective spin model becomes

$$
\mathcal{H}_{\mathrm{LGT}}=-K \sum_{\bigcirc_{\mathrm{d}}} \cos (\operatorname{curl} A)+U \sum_{\boldsymbol{r} \boldsymbol{r}^{\prime}}\left(E_{\boldsymbol{r} \boldsymbol{r}^{\prime}}-\frac{\eta_{\boldsymbol{r}}}{2}\right)^{2},
$$

where $K=24 J_{\perp}^{3} / J_{z z}^{2}$ and " $U \rightarrow \infty$ " recovers the Hilbert space of the spin-1/2 moment. Here " $\square_{\mathrm{d}}$ " refers to the elementary hexagon on the diamond lattice, and $\eta_{\boldsymbol{r}}=+1$ $(-1)$ for $\boldsymbol{r} \in \mathrm{I}$ (II) sublattice of the diamond lattice. When $J_{\perp}>0$ and $\left|J_{\perp}\right|$ is small so that the XY order is absent, the ground state favors a zero $\mathrm{U}(1)$ gauge flux and is labeled as $\mathrm{U}(1)_{0}$ QSL. This regime has been extensively studied theoretically and numerically ${ }^{1,8,9,28,49-51}$. For $J_{\perp}<0$, the ground state favors a $\pi$ background $\mathrm{U}(1)$ gauge flux with ${ }^{9}$

$$
\operatorname{curl} A \equiv \sum_{\boldsymbol{r} \boldsymbol{r}^{\prime} \in \square_{\mathrm{d}}} A_{\boldsymbol{r} \boldsymbol{r}^{\prime}}=\pi
$$

for each diamond lattice hexagon (see Fig. 2a) and is thus labeled as $\mathrm{U}(1)_{\pi}$ QSL. This regime has a sign problem for quantum Monte Carlo simulation and is thus less explored. Only one prior work ${ }^{9}$ has carefully studied the stability of the U(1) QSL in this regime and found the $\mathrm{U}(1)$ QSL is more robust in this regime than the $J_{\perp}>0$ regime. Despite the different phase stability, both $\mathrm{U}(1)_{0}$ and $\mathrm{U}(1)_{\pi}$ QSLs are described by the same low-energy field theory and characterized by the same long-distance universal properties. We, however, point out that the $\mathrm{U}(1)_{\pi}$ QSL is a distinct symmetry enriched U(1) QSL from the $\mathrm{U}(1)_{0}$ QSL. We show below that the symmetry enrichment occurs in the translational symmetry fractionalization of the spinons. We emphasize that the spectral periodicity of the spinon continuum is a keen physical property encoding the distinct symmetry enrichment and could thus provide a sharp experimental confirmation of the U(1) QSL.

\section{TRANSLATIONAL SYMMETRY FRACTIONALIZATION AND THE SPECTRAL PERIODICITY}

The translation symmetry of the pyrochlore lattice is generated by the three translations $T_{1}, T_{2}$, and $T_{3}$. Here, the $T_{\mu}$ operation translates the system by the fcc bravais lattice vector $\boldsymbol{a}_{\mu}$, and we have $\boldsymbol{a}_{1}=\frac{1}{2}(011), \boldsymbol{a}_{2}=\frac{1}{2}(101)$, and $\boldsymbol{a}_{3}=\frac{1}{2}(110)$, where we have used the cubic coordinate system here and throughout the paper (except specifically mentioned). Any two translation operations, $T_{\mu}$ and $T_{\nu}(\mu \neq \nu)$, commute with each other with $T_{\mu} T_{\nu}=T_{\nu} T_{\mu}$.

In the U(1) QSL, the spinons are fractionalized and deconfined excitations, and the symmetry operations act locally on the spinons. This symmetry localization condition leads to the symmetry fractionalization for the spinons. For the translation symmetry under consideration, we have

$$
T_{\mu}^{s} T_{\nu}^{s}= \pm T_{\nu}^{s} T_{\mu}^{s}
$$




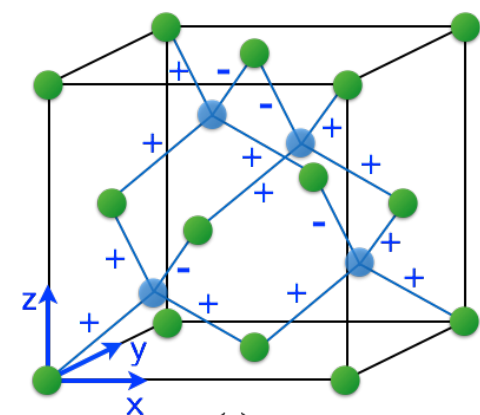

(a)

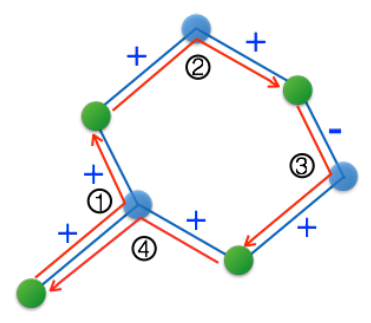

(b)

FIG. 2. (Color online.) The diamond lattice formed by the tetrahedral centers of the pyrochlore lattice. The dots are the diamond lattice sites or the tetrahedral centers of the pyrochlore lattice. (a) The spinon hopping for a specific gauge choice for the $\pi$ flux. (b) The successive translations of the spinon along the (red) pathway, that are marked by (1), (2), (3) and (4), experience the U(1) gauge flux in the hexagon plaquette.

where $T_{\mu}^{s}, T_{\nu}^{s}$ are the translation operators that operate on the individual spinon. The time reversal symmetry demands that $T_{\mu}^{s}$ and $T_{\nu}^{s}$ either commute or anticommute with each other. As the spinon tunnels on the lattice successively following the translation operation $T_{\mu}^{s} T_{\nu}^{s}\left(T_{\mu}^{s}\right)^{-1}\left(T_{\nu}^{s}\right)^{-1}$, the spinon experiences the background $\mathrm{U}(1)$ gauge flux. If the background $\mathrm{U}(1)$ gauge flux is $0(\pi)$, "+" ("-") sign is chosen in Eq. (5).

For the XXZ model, it was shown that, in the regime with $J_{\perp}<0$, each elementary hexagon plaquette of the diamond lattice formed by the tetrahedral centers traps a $\pi \mathrm{U}(1)$ gauge flux for the spinon ${ }^{1}$. The spinons are created in pairs by the spin flipping operators $S^{ \pm}$and reside on the diamond lattice sites of the neighboring tetrahedral centers. It is ready to see from the (red) path on the diamond lattice in Fig. $2 \mathrm{~b}$ that transporting the spinon according to $T_{\mu}^{s} T_{\nu}^{s}\left(T_{\mu}^{s}\right)^{-1}\left(T_{\nu}^{s}\right)^{-1}$ experiences the same gauge flux in the elementary hexagon plaquette. Therefore, for the $\mathrm{U}(1)_{\pi}$ QSL with $J_{\perp}<0$, we have $T_{\mu}^{s} T_{\nu}^{s}=-T_{\nu}^{s} T_{\mu}^{s}$. In comparison, for the U(1) 0 QSL with $J_{\perp}>0$, we have $T_{\mu}^{s} T_{\nu}^{s}=+T_{\nu}^{s} T_{\mu}^{s}$.

We now explore the sepctroscopic consequence of the non-trivial translational symmmetry fractionalization for the spinons in the $\mathrm{U}(1)_{\pi}$ QSL. To reveal the property of the spinon continuum, we consider a generic two-spinon scattering state ${ }^{46,48,52}$

$$
|a\rangle \equiv\left|\boldsymbol{q}_{a} ; z_{a}\right\rangle,
$$

where $\boldsymbol{q}_{a}$ labels the total crystal momentum and $z_{a}$ refers to the remaining quantum numbers such as the total energy of the state. Due to the non-orthogonality of the fcc bravais lattice vectors, for our convenience we express the momentum $\boldsymbol{q}_{a}$ as

$$
\boldsymbol{q}_{a}=q_{a 1} \boldsymbol{e}_{1}+q_{a 2} \boldsymbol{e}_{2}+q_{a 3} \boldsymbol{e}_{3},
$$

where $\boldsymbol{e}_{1}=(-1,1,1), \boldsymbol{e}_{2}=(1,-1,1), \boldsymbol{e}_{3}=(1,1,-1)$, and $q_{a 1}, q_{a 2}, q_{a 3}$ are the projection of $\boldsymbol{q}_{a}$ onto the cor-

responding $\boldsymbol{e}$ vectors. From the symmetry localization condition for the spinons, the lattice translation $T_{\mu}$ acts on the state as

$$
T_{\mu}|a\rangle=T_{\mu}^{s}(1) T_{\mu}^{s}(2)|a\rangle,
$$

where ' 1 ' and ' 2 ' label the two spinons, and the translation is "decomposed" into the two spinon translations. In the following, we apply the approach that was developed for the $2 \mathrm{D} \mathbb{Z}_{2}$ QSL in Ref. 48, but adapt the discussion to our 3D U(1) $)_{\pi}$ QSL. We apply the spinon translation on the spinon 1 of the state $|a\rangle$ to generate the other three two-spinon scattering states,

$$
\begin{aligned}
|b\rangle & =T_{1}^{s}(1)|a\rangle, \\
|c\rangle & =T_{2}^{s}(1)|a\rangle, \\
|d\rangle & =T_{3}^{s}(1)|a\rangle .
\end{aligned}
$$

All the above states are energy eigenstates and have the same energy as the two-spinon scattering state $|a\rangle$. Nevertheless, these spinon scattering states have distinct crystal momenta. To show that, we apply the translation operations on the state $|b\rangle$,

$$
\begin{aligned}
& T_{1}|b\rangle=T_{1}^{s}(1) T_{1}^{s}(2) T_{1}^{s}(1)|a\rangle=+T_{1}^{s}(1)\left[T_{1}|a\rangle\right], \\
& T_{2}|b\rangle=T_{2}^{s}(1) T_{2}^{s}(2) T_{1}^{s}(1)|a\rangle=-T_{1}^{s}(1)\left[T_{2}|a\rangle\right], \\
& T_{3}|b\rangle=T_{3}^{s}(1) T_{3}^{s}(2) T_{1}^{s}(1)|a\rangle=-T_{1}^{s}(1)\left[T_{3}|a\rangle\right],
\end{aligned}
$$

where the anticommutation relation between two spinon translations are used in the last two equations. This immediately gives

$$
q_{b 1}=q_{a 1}, \quad q_{b 2}=q_{a 2}+\pi, \quad q_{b 3}=q_{a 3}+\pi .
$$

Likewise, we have

$$
\begin{array}{ll}
q_{c 1}=q_{a 1}+\pi, & q_{c 2}=q_{a 2}, \quad q_{c 3}=q_{a 3}+\pi, \\
q_{d 1}=q_{a 1}+\pi, & q_{d 2}=q_{a 2}+\pi, \quad q_{d 3}=q_{a 3} .
\end{array}
$$

The combination of two different spinon translations on $|a\rangle$ such as $T_{1}^{s}(1) T_{2}^{s}(1)|a\rangle$ does not generate new states with different momenta. Since the two-spinon scattering states, $|a\rangle,|b\rangle,|c\rangle,|d\rangle$, have the same energy and the same spin quantum number, the above relations between their crystal momenta suggest that, there is an enhanced spectral periodicity for the spinon continuum. The spectral periodicity can be reflected by the lower $\mathcal{L}(\boldsymbol{q})$, upper excitation edge $\mathcal{U}(\boldsymbol{q})$, and the dispersion of the spinon continuum. For the $\mathrm{U}(1)_{\pi}$ QSL, we have

$$
\begin{aligned}
\mathcal{L}(\boldsymbol{q}) & =\mathcal{L}(\boldsymbol{q}+2 \pi(100))=\mathcal{L}(\boldsymbol{q}+2 \pi(010)) \\
& =\mathcal{L}(\boldsymbol{q}+2 \pi(001)), \\
\mathcal{U}(\boldsymbol{q}) & =\mathcal{U}(\boldsymbol{q}+2 \pi(100))=\mathcal{U}(\boldsymbol{q}+2 \pi(010)) \\
& =\mathcal{U}(\boldsymbol{q}+2 \pi(001)),
\end{aligned}
$$

where the momentum and the momentum offset are defined in the cubic coordinate system. The spectral intensity of the spinon continnum depends on other factors such as the form factor and may not respect the enhanced spectral periodicity here. 
Usually, the spectral periodicity of the magnetic excitation spectrum is defined by the integer mutiples of the reciprocal lattice vectors. Here, because of the $\pi$ flux and the translational symmetry fractionalization for the $\mathrm{U}(1)_{\pi}$ QSL, the Brillouin zone is folded and the spectral periodicity is half of the combination of two indepedent reciprocal lattice vectors. The spectral periodicity enhancement is a rather unique property of the $\mathrm{U}(1)_{\pi}$ QSL and is absent in the $\mathrm{U}(1)_{0}$ QSL. We emphasize that the enhanced spectral periodicity with a folded Brillouin zone is the dynamical property rather than the static property of the $\mathrm{U}(1)_{\pi}$ QSL. The $\mathrm{U}(1)_{\pi}$ QSL preserves all the lattice symmetries, and an elastic neutron scattering would not observe any extra magnetic Bragg peak that accompanys with any lattice symmetry breaking.

\section{SPINON CONTINUUM OF THE U(1) $)_{\pi}$ QSL}

Here we return to the specific XXZ model and explicitly demonstrate the experimental consequence of the background $\pi$ flux in the $\mathrm{U}(1)_{\pi}$ QSL. We focus on the $J_{\perp}<0$ regime that has not been extensively studied. It was shown that the $\mathrm{U}(1)_{\pi}$ QSL extends to the point ${ }^{9}$ at $J_{\perp}=-4.13 J_{z z}$ within a gauge mean-field calculation. We, however, do not think the $\mathrm{U}(1)_{\pi}$ QSL can extend beyond the Heisenberg point at $J_{\perp}=-J_{z z} / 2$ where the $\mathrm{SU}(2)$ symmetry, that permutes the spin components, is inconsistent with the distinct physical meaning of three spin components in the $\mathrm{U}(1)_{\pi}$ QSL. It is likely that the Heisenberg point is a critical point where the $\mathrm{U}(1)_{\pi}$ QSL terminates. Nevertheless, the early study does show the quantitative stability of the $\mathrm{U}(1)_{\pi}$ QSL. Following the previous treatment ${ }^{6,8-10}$, we implement the spinon-gauge construction via

$$
\begin{aligned}
S_{i}^{z} & =s_{\boldsymbol{r} \boldsymbol{r}^{\prime}}^{z}, \\
S_{i}^{+} & =\Phi_{\boldsymbol{r}}^{\dagger} \Phi_{\boldsymbol{r}^{\prime}} s_{\boldsymbol{r} \boldsymbol{r}^{\prime}}^{+},
\end{aligned}
$$

where $\Phi_{r}^{\dagger}\left(\Phi_{\boldsymbol{r}}\right)$ creates (annihilates) the spinon at the diamond lattice site $\boldsymbol{r}$, and $s^{z}$ and $s^{ \pm}$encode the U(1) gauge field such that $s_{\boldsymbol{r} \boldsymbol{r}^{\prime}}^{z} \simeq E_{\boldsymbol{r} \boldsymbol{r}^{\prime}}$ and $s_{\boldsymbol{r} \boldsymbol{r}^{\prime}}^{+} \simeq \frac{1}{2} e^{i A_{\boldsymbol{r} \boldsymbol{r}^{\prime}}}$. The $\mathrm{XXZ}$ model is expressed as

$$
H_{\mathrm{XXZ}} \simeq \frac{J_{z z}}{2} \sum_{\boldsymbol{r}} Q_{\boldsymbol{r}}^{2}-\frac{J_{\perp}}{4} \sum_{\left\langle\left\langle\boldsymbol{r} \boldsymbol{r}^{\prime}\right\rangle\right\rangle} \Phi_{\boldsymbol{r}}^{\dagger} \Phi_{\boldsymbol{r}^{\prime}} e^{-i A_{\boldsymbol{r} \boldsymbol{r}^{\prime}}}
$$

where $A_{\boldsymbol{r} \boldsymbol{r}^{\prime}}=A_{\boldsymbol{r} \boldsymbol{r}^{\prime \prime}}+A_{\boldsymbol{r}^{\prime \prime} \boldsymbol{r}^{\prime}}$, and $\boldsymbol{r}^{\prime \prime}$ is the shared nearest neigbhor site of $\boldsymbol{r}$ and $\boldsymbol{r}^{\prime}$. Here the operator $Q_{\boldsymbol{r}}$ is defined as $Q_{\boldsymbol{r}}=\sum_{\boldsymbol{r}^{\prime} \in n . n .(\boldsymbol{r})} \eta_{\boldsymbol{r}} S_{\boldsymbol{r} \boldsymbol{r}^{\prime}}^{z}$, where the summation is taken for the nearest neighbor sites of $\boldsymbol{r}$. A conjugate rotor variable is introduced such that

$$
\Phi_{\boldsymbol{r}}=e^{-i \phi_{\boldsymbol{r}}}, \quad\left|\Phi_{\boldsymbol{r}}\right|=1
$$

and $\left[\phi_{\boldsymbol{r}}, Q_{\boldsymbol{r}}\right]=i$. One further fixes the gauge by setting $^{9} \bar{A}_{\boldsymbol{r} \boldsymbol{r}^{\prime}}=\epsilon_{\boldsymbol{r} \boldsymbol{r}^{\prime}} \boldsymbol{q}_{0} \cdot \boldsymbol{r}$ that takes care of the $\pi$ flux (see Fig. 2a), where $\boldsymbol{q}_{0}=2 \pi(100), \boldsymbol{r} \in \mathrm{I}$ sublattice, and $\epsilon_{\boldsymbol{r} \boldsymbol{r}^{\prime}}$ takes the value $0,1,1,0$ for $\boldsymbol{r} \boldsymbol{r}^{\prime}$ orienting along (111), (a)
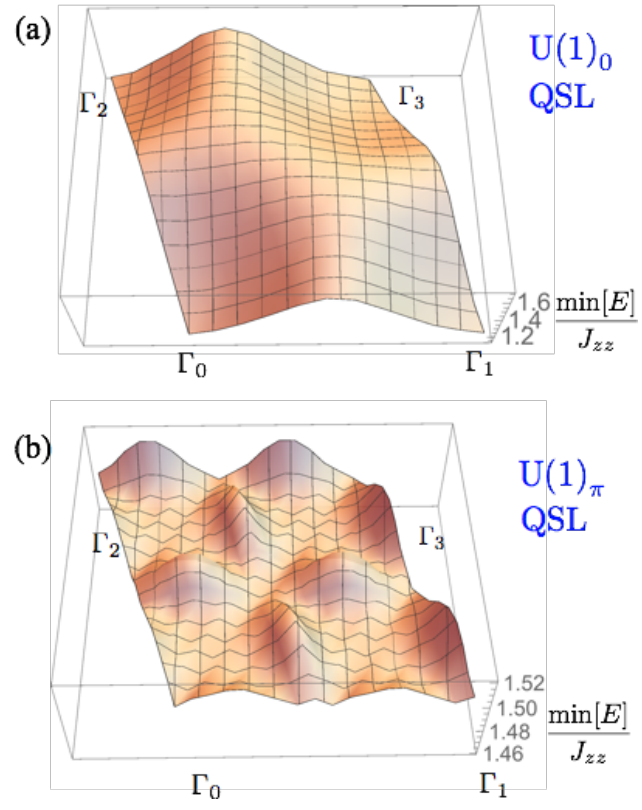

FIG. 3. (Color online.) The lower excitation edge of the spinon continuum in the $\mathrm{U}(1)_{0}$ and the $\mathrm{U}(1)_{\pi}$ QSLs. Here, the $\Gamma$ points are the centers of the Brillouin zones and are connected by the reciprocal lattice vectors with $\Gamma_{0} \Gamma_{1}=$ $2 \pi(-1,1,1)$ and $\Gamma_{0} \Gamma_{2}=2 \pi(1,-1,1)$. The enhanced spectral periodicity in (b) can be visualized by examining the wiggles of the spectrum. We set $J_{\perp}=0.12 J_{z z}$ for the $\mathrm{U}(1)_{0}$ QSL in (a) and $J_{\perp}=-J_{z z} / 3$ for the $\mathrm{U}(1)_{\pi}$ QSL in (b).

$(1 \overline{1} \overline{1}),(\overline{1} 1 \overline{1}),(\overline{1} \overline{1} 1)$ lattice direction, respectively. The gauge fixing condition enlarges the unit cell for the spinons, but the translation symmetry is preserved and is realized projectively. The spinon excitation in $\mathrm{U}(1)_{\pi}$ QSL is then solved by the standard coherent state path integral method and is given as ${ }^{9}$

$$
\begin{aligned}
\omega_{\mathrm{I}, \pm}(\boldsymbol{k}) & =\sqrt{2 J_{z z}\left(\lambda \pm J_{\perp}\left(c_{y}^{2} c_{z}^{2}+s_{x}^{2} s_{y}^{2}+c_{x}^{2} s_{z}^{2}\right)^{\frac{1}{2}}\right)}, \\
\omega_{\mathrm{II}, \pm}(\boldsymbol{k}) & =\sqrt{2 J_{z z}\left(\lambda \pm J_{\perp}\left(s_{y}^{2} s_{z}^{2}+c_{x}^{2} c_{y}^{2}+s_{x}^{2} c_{z}^{2}\right)^{\frac{1}{2}}\right)},
\end{aligned}
$$

where $c_{\mu}=\cos \left(k_{\mu} / 2\right), s_{\mu}=\sin \left(k_{\mu} / 2\right)$. The subindices, I, II, arise from the fact that the two diamond lattices are decoupled in Eq. (22) and the subindices, \pm , arise from the doubling of the unit cell by the gauge choice. Here, the constraint $\left|\Phi_{\boldsymbol{r}}\right|=1$ is implemented by the global Lagrangian multiplier, $\lambda$, that is demanded to be uniform for the two sublattices by inversion.

The spinon continuum is detected by the $\left\langle S_{i}^{+} S_{j}^{-}\right\rangle$correlator via the INS. From the relation

$$
\begin{aligned}
\left\langle S_{i}^{+} S_{j}^{-}\right\rangle & \sim\left\langle\Phi_{\boldsymbol{r}_{i}}^{\dagger} \Phi_{\boldsymbol{r}_{i}^{\prime}} e^{i A_{\boldsymbol{r}_{i} \boldsymbol{r}_{i}^{\prime}}} \Phi_{\boldsymbol{r}_{j}} \Phi_{\boldsymbol{r}_{j}^{\prime}}^{\dagger} e^{-i A_{\boldsymbol{r}_{i} \boldsymbol{r}_{j}^{\prime}}}\right\rangle \\
& \simeq\left\langle\Phi_{\boldsymbol{r}_{i}}^{\dagger} \Phi_{\boldsymbol{r}_{j}}\right\rangle\left\langle\Phi_{\boldsymbol{r}_{i}^{\prime}} \Phi_{\boldsymbol{r}_{j}^{\prime}}^{\dagger}\right\rangle\left\langle e^{i \bar{A}_{\boldsymbol{r}_{i} \boldsymbol{r}_{i}^{\prime}}-i \bar{A}_{\boldsymbol{r}_{i} \boldsymbol{r}_{j}^{\prime}}}\right\rangle,
\end{aligned}
$$

where $\boldsymbol{r}_{i}, \boldsymbol{r}_{j} \in \mathrm{I}, \boldsymbol{r}_{i}^{\prime}, \boldsymbol{r}_{j}^{\prime} \in \mathrm{II}$, and the neutron spin flip excites two spinons with one from the I sublattice and the other from the II sublattice, we obtain the momentum 
and energy transfers of the neutron,

$$
\begin{aligned}
\boldsymbol{q} & =\boldsymbol{k}_{1}+\boldsymbol{k}_{2}+\boldsymbol{q}_{0}, \\
E & =\omega_{\mathrm{I}, \mu}\left(\boldsymbol{k}_{1}\right)+\omega_{\mathrm{II}, \nu}\left(\boldsymbol{k}_{2}\right),
\end{aligned}
$$

where $\mu, \nu= \pm$ and the offset $\boldsymbol{q}_{0}$ arises from the particular gauge choice for the $\mathrm{U}(1)_{\pi}$ QSL, and the predicted physical observable does not depend on this choice. Here we have neglected the photon contribution that appears as a higher order term from the gauge fluctuation with respect to the gauge choice in the expansion of Eq. (26). The spinons are gapped, and a minimal energy is required to excite them, which defines the lower excitation edge. As we show explicitly in Fig. 3, the lower excitation edge of the $\mathrm{U}(1)_{\pi}$ QSL has the enhanced periodicity while the $\mathrm{U}(1)_{0}$ QSL does not.

\section{DISCUSSION}

Although the gapless $\mathrm{U}(1)$ gauge photon is one defining feature of the U(1) QSLs, its very-low-energy scale and the suppressed spectral weight may prohibit the experimental identification ${ }^{8,36}$. In contrast, the spinon continuum occurs at the higher energy. The enhanced spectral periodicity with a fold Brillouin zone of the spinon continuum in the $\mathrm{U}(1)_{\pi}$ QSL could be a sharp signature for the experimental observation. Since the U(1) $)_{\pi}$ QSL occupies a larger parameter space than the $\mathrm{U}(1)_{0} \mathrm{QSL}^{9}$, it is thus more likely for a candidate system to locate in the $\mathrm{U}(1)_{\pi}$ QSL and develop the enhanced spectral periodicity that we predict for the spinon continuum.

There are three types of doublets in the rare-earth pyrochlore systems. For the non-Kramers doublet like $\mathrm{Pr}^{3+}$ in $\mathrm{Pr}_{2} \mathrm{Ir}_{2} \mathrm{O}_{7}$ and $\mathrm{Pr}_{2} \mathrm{Zr}_{2} \mathrm{O}_{7}^{7,9,31,53}$ since only the Ising component of the local moment is odd under the time reversal, the INS would naturally select the Ising components and hence only measure the gauge field correlator. The spinon continuum cannot be observed for the non-Kramers doublet. For the usual Kramers doublet like $\mathrm{Yb}^{3+}$ in $\mathrm{Yb}_{2} \mathrm{Ti}_{2} \mathrm{O}_{7}{ }^{8,10,11,27}$, all the components contribute to the magnetic dipolar moments and are thus visible in the INS measurements. Both the gapped spinon continuum and the gapless gauge photon are recorded in the INS spectrum. As for the dipole-octupole Kramers doublet like $\mathrm{Ce}^{3+}$ in $\mathrm{Ce}_{2} \mathrm{Sn}_{2} \mathrm{O}_{7}{ }^{12,14,41,54}$, it was predicted that $^{12,14}$, two distinct symmetry enriched U(1) QSLs, namely, the dipolar U(1) QSL and the octupolar U(1) QSL, can occur. For the dipolar U(1) QSL, both the gapped spinon continuum and the gapless gauge photon show up in the INS spectrum, while for the octupolar U(1) QSL, only gapped spinon continuum can be detected by the INS ${ }^{14}$. Therefore, we suggest a careful examination of the spectral periodicity of the spinon continuum for the QSI materials with Kramers doublets.

Besides the spectral periodicity, the proximate magnetic order provides indirect information about the nearby QSLs. Due to the background $\pi$ flux, we expect the proximate magnetic order of the $\mathrm{U}(1)_{\pi}$ QSL generically breaks the translation symmetry, while the proximate $\mathrm{AFM}_{0}$ state of the U(1) $)_{0}$ QSL does not ${ }^{8}$. For the non-Kramers doublets, the spinon condensation from the $\mathrm{U}(1)_{\pi}$ QSL leads to the transverse spin order that preserves the time reversal and corresponds to the magnetic quadrupolar order ${ }^{7,9}$. Although the elastic neutron diffraction may not directly probe the quadrupolar order and the enlargement of the unit cell, the spinwave excitation, that is created by the dipolar component and detected by INS, reveals the intrinsic quadrupolar order and the translation symmetry breaking. For the Kramers doublets, the transverse spin order is concomitant with the time reversal symmetry breaking and is readily detected by neutron diffraction and/or NMR measurements.

\section{ACKNOWLEDGEMENTS}

I think the anonymous referees for suggestion that improves the presentation of this paper. I acknowledge Dr. Jeffrey Rau from University of Waterloo for a conversation and Professor Michel Gingras for a comment. I am deeply indebted to Professor Dunghai Lee whose advice and encouragement motivated me to write out and submit my papers including this one. This work is supported by the Ministry of Science and Technology of China with the Grant No.2016YFA0301001, the StartUp Funds and the Program of First-Class Construction of Fudan University, and the Thousand-Youth-Talent Program of China.
* gangchen.physics@gmail.com

1 Michael Hermele, Matthew P. A. Fisher, and Leon Balents, "Pyrochlore photons: The $U(1)$ spin liquid in a $S=\frac{1}{2}$ three-dimensional frustrated magnet," Phys. Rev. B 69, 064404 (2004).

2 O. I. Motrunich and T. Senthil, "Origin of artificial electrodynamics in three-dimensional bosonic models," Phys. Rev. B 71, 125102 (2005).
3 David A. Huse, Werner Krauth, R. Moessner, and S. L. Sondhi, "Coulomb and Liquid Dimer Models in Three Dimensions," Phys. Rev. Lett. 91, 167004 (2003).

4 Hamid R. Molavian, Michel J. P. Gingras, and Benjamin Canals, "Dynamically Induced Frustration as a Route to a Quantum Spin Ice State in $\mathrm{Tb}_{2} \mathrm{Ti}_{2} \mathrm{O}_{7}$ via Virtual Crystal Field Excitations and Quantum Many-Body Effects," Phys. Rev. Lett. 98, 157204 (2007). 
${ }^{5}$ M J P Gingras and P A McClarty, "Quantum spin ice: a search for gapless quantum spin liquids in pyrochlore magnets," Reports on Progress in Physics 77, 056501 (2014).

6 Lucile Savary and Leon Balents, "Quantum spin liquids: a review," Reports on Progress in Physics 80, 016502 (2016).

7 Shigeki Onoda and Yoichi Tanaka, "Quantum Melting of Spin Ice: Emergent Cooperative Quadrupole and Chirality," Phys. Rev. Lett. 105, 047201 (2010).

8 Lucile Savary and Leon Balents, "Coulombic Quantum Liquids in Spin-1/2 Pyrochlores," Phys. Rev. Lett. 108, 037202 (2012).

9 SungBin Lee, Shigeki Onoda, and Leon Balents, "Generic quantum spin ice," Phys. Rev. B 86, 104412 (2012).

10 Lucile Savary and Leon Balents, "Spin liquid regimes at nonzero temperature in quantum spin ice," Phys. Rev. B 87, 205130 (2013).

11 K. A. Ross, J. P. C. Ruff, C. P. Adams, J. S. Gardner, H. A. Dabkowska, Y. Qiu, J. R. D. Copley, and B. D. Gaulin, "Two-Dimensional Kagome Correlations and Field Induced Order in the Ferromagnetic $X Y$ Pyrochlore $\mathrm{Yb}_{2} \mathrm{Ti}_{2} \mathrm{O}_{7}$," Phys. Rev. Lett. 103, 227202 (2009).

12 Yi-Ping Huang, Gang Chen, and Michael Hermele, "Quantum Spin Ices and Topological Phases from DipolarOctupolar Doublets on the Pyrochlore Lattice," Phys. Rev. Lett. 112, 167203 (2014).

13 Yuan Wan and Oleg Tchernyshyov, "Quantum Strings in Quantum Spin Ice," Phys. Rev. Lett. 108, 247210 (2012).

14 Yao-Dong Li and Gang Chen, "Symmetry enriched U(1) topological orders for dipole-octupole doublets on a pyrochlore lattice," Phys. Rev. B 95, 041106 (2017).

15 Han Yan, Owen Benton, Ludovic Jaubert, and Nic Shannon, "Theory of multiple-phase competition in pyrochlore magnets with anisotropic exchange with application to $\mathrm{Yb}_{2} \mathrm{Ti}_{2} \mathrm{O}_{7}, \mathrm{Er}_{2} \mathrm{Ti}_{2} \mathrm{O}_{7}$, and $\mathrm{Er}_{2} \mathrm{Sn}_{2} \mathrm{O}_{7}$," Phys. Rev. B 95, 094422 (2017).

16 Lucile Savary, Xiaoqun Wang, Hae-Young Kee, Yong Baek Kim, Yue $\mathrm{Yu}$, and Gang Chen, "Quantum spin ice on the breathing pyrochlore lattice," Phys. Rev. B 94, 075146 (2016).

17 T. Fennell, M. Kenzelmann, B. Roessli, M. K. Haas, and R. J. Cava, "Power-Law Spin Correlations in the Pyrochlore Antiferromagnet $\mathrm{Tb}_{2} \mathrm{Ti}_{2} \mathrm{O}_{7}$," Phys. Rev. Lett. 109, 017201 (2012).

18 Yukio Yasui, Masaki Kanada, Masafumi Ito, Hiroshi Harashina, Masatoshi Sato, Hajime Okumura, Kazuhisa Kakurai, and Hiroaki Kadowaki, "Static Correlation and Dynamical Properties of $\mathrm{Tb}^{3+}$-moments in $\mathrm{Tb}_{2} \mathrm{Ti}_{2} \mathrm{O}_{7}$ Neutron Scattering Study," Journal of the Physical Society of Japan 71, 599-606 (2002).

19 J. S. Gardner, B. D. Gaulin, A. J. Berlinsky, P. Waldron, S. R. Dunsiger, N. P. Raju, and J. E. Greedan, "Neutron scattering studies of the cooperative paramagnet pyrochlore $\mathrm{Tb}_{2} \mathrm{Ti}_{2} \mathrm{O}_{7}$," Phys. Rev. B 64, 224416 (2001).

${ }^{20}$ Zhihao Hao, Alexandre G. R. Day, and Michel J. P. Gingras, "Bosonic many-body theory of quantum spin ice," Phys. Rev. B 90, 214430 (2014).

21 Lieh-Jeng Chang, Shigeki Onoda, Yixi Su, Ying-Jer Kao, Ku-Ding Tsuei, Yukio Yasui, Kazuhisa Kakurai, and Martin Richard Lees, "Higgs transition from a magnetic Coulomb liquid to a ferromagnet in $\mathrm{Yb}_{2} \mathrm{Ti}_{2} \mathrm{O}_{7}$, , Nature Communications 3, 992 (2012).

${ }^{22}$ K. Kimura, K. Nakatsuji, J-J. Wen, C. Broholm, M.B. Stone, E. Nishibori, and H. Sawa, "Quantum fluctuations in spin-ice-like $\mathrm{Pr}_{2} \mathrm{Zr}_{2} \mathrm{O}_{7}$," Nature Communications
4, 2914 (2013)

23 Jason S. Gardner, Michel J. P. Gingras, and John E. Greedan, "Magnetic pyrochlore oxides," Rev. Mod. Phys. 82, 53-107 (2010).

24 E. Lhotel, S. R. Giblin, M. R. Lees, G. Balakrishnan, L. J. Chang, and Y. Yasui, "First-order magnetic transition in $\mathrm{Yb}_{2} \mathrm{Ti}_{2} \mathrm{O}_{7}$," Phys. Rev. B 89, 224419 (2014).

25 Lieh-Jeng Chang, Martin R. Lees, Isao Watanabe, Adrian D. Hillier, Yukio Yasui, and Shigeki Onoda, "Static magnetic moments revealed by muon spin relaxation and thermodynamic measurements in the quantum spin ice $\mathrm{Yb}_{2} \mathrm{Ti}_{2} \mathrm{O}_{7}$," Phys. Rev. B 89, 184416 (2014).

26 Yukio Yasui, Minoru Soda, Satoshi Iikubo, Masafumi Ito, Masatoshi Sato, Nobuko Hamaguchi, Taku Matsushita, Nobuo Wada, Tetsuya Takeuchi, Naofumi Aso, and Kazuhisa Kakurai, "Ferromagnetic Transition of Pyrochlore Compound $\mathrm{Yb}_{2} \mathrm{Ti}_{2} \mathrm{O}_{7}$," Journal of the Physical Society of Japan 72, 3014-3015 (2003).

27 Kate Ross, Lucile Savary, Bruce Gaulin, and Leon Balents, "Quantum Excitations in Quantum Spin Ice," Phys. Rev. X 1, 021002 (2011).

28 Nic Shannon, Olga Sikora, Frank Pollmann, Karlo Penc, and Peter Fulde, "Quantum Ice: A Quantum Monte Carlo Study," Phys. Rev. Lett. 108, 067204 (2012).

29 Pallab Goswami, Bitan Roy, and Sankar Das Sarma, "Itinerant spin ice order, Weyl metal, and anomalous Hall effect in $\mathrm{Pr}_{2} \mathrm{Ir}_{2} \mathrm{O}_{7}, "$ ArXiv:1603.02273 (2016).

${ }^{30}$ K. E. Arpino, B. A. Trump, A. O. Scheie, T. M. McQueen, and S. M. Koohpayeh, "Impact of stoichiometry of $\mathrm{Yb}_{2} \mathrm{Ti}_{2} \mathrm{O}_{7}$ on its physical properties," Phys. Rev. B 95, 094407 (2017).

31 Gang Chen, "Magnetic monopole" condensation of the pyrochlore ice $\mathrm{U}(1)$ quantum spin liquid: Application to $\mathrm{Pr}_{2} \mathrm{Ir}_{2} \mathrm{O}_{7}$ and $\mathrm{Yb}_{2} \mathrm{Ti}_{2} \mathrm{O}_{7}$," Phys. Rev. B 94, 205107 (2016).

32 J.-J. Wen, S. M. Koohpayeh, K. A. Ross, B. A. Trump, T. M. McQueen, K. Kimura, S. Nakatsuji, Y. Qiu, D. M. Pajerowski, J. R. D. Copley, and C. L. Broholm, "Disordered Route to the Coulomb Quantum Spin Liquid: Random Transverse Fields on Spin Ice in $\mathrm{Pr}_{2} \mathrm{Zr}_{2} \mathrm{O}_{7}$," Phys. Rev. Lett. 118, 107206 (2017).

33 Gang Chen, "What does inelastic neutron scattering measure in quantum spin ices?" ArXiv:1706.04333 (2017).

34 D. E. MacLaughlin, O. O. Bernal, Lei Shu, Jun Ishikawa, Yosuke Matsumoto, J.-J. Wen, M. Mourigal, C. Stock, G. Ehlers, C. L. Broholm, Yo Machida, Kenta Kimura, Satoru Nakatsuji, Yasuyuki Shimura, and Toshiro Sakakibara, "Unstable spin-ice order in the stuffed metallic pyrochlore $\operatorname{Pr}_{2+x} \operatorname{Ir}_{2-x} \mathrm{O}_{7-\delta}$," Phys. Rev. B 92, 054432 (2015).

35 Jianlong Fu, Jeffrey G Rau, Michel JP Gingras, and Natalia B Perkins, "Fingerprints of quantum spin ice in raman scattering," arXiv preprint arXiv:1703.03836 (2017).

36 Owen Benton, Olga Sikora, and Nic Shannon, "Seeing the light: Experimental signatures of emergent electromagnetism in a quantum spin ice," Phys. Rev. B 86, 075154 (2012).

37 L. D. C. Jaubert, Owen Benton, Jeffrey G. Rau, J. Oitmaa, R. R. P. Singh, Nic Shannon, and Michel J. P. Gingras, "Are Multiphase Competition and Order by Disorder the Keys to Understanding $\mathrm{Yb}_{2} \mathrm{Ti}_{2} \mathrm{O}_{7}$ ?" Phys. Rev. Lett. 115, 267208 (2015).

38 R. Applegate, N. R. Hayre, R. R. P. Singh, T. Lin, A. G. R. Day, and M. J. P. Gingras, "Vindication of $\mathrm{Yb}_{2} \mathrm{Ti}_{2} \mathrm{O}_{7}$ as a Model Exchange Quantum Spin Ice," Phys. Rev. Lett. 
109, 097205 (2012).

39 Mathieu Taillefumier, Owen Benton, Han Yan, Ludovic Jaubert, and Nic Shannon, "Frustrating quantum spin ice: a tale of three spin liquids, and hidden order," ArXiv:1705.00148 (2017).

40 S. R. Dunsiger, A. A. Aczel, C. Arguello, H. Dabkowska, A. Dabkowski, M.-H. Du, T. Goko, B. Javanparast, T. Lin, F. L. Ning, H. M. L. Noad, D. J. Singh, T. J. Williams, Y. J. Uemura, M. J. P. Gingras, and G. M. Luke, "Spin ice: Magnetic excitations without monopole signatures using muon spin rotation," Phys. Rev. Lett. 107, 207207 (2011).

41 Romain Sibille, Elsa Lhotel, Vladimir Pomjakushin, Chris Baines, Tom Fennell, and Michel Kenzelmann, "Candidate Quantum Spin Liquid in the $\mathrm{Ce}^{3+}$ Pyrochlore Stannate $\mathrm{Ce}_{2} \mathrm{Sn}_{2} \mathrm{O}_{7}$," Phys. Rev. Lett. 115, 097202 (2015).

42 For the XXZ model, $\mathrm{AFM}_{0}$ is a ferromagnetic state. But in the local coordinate system for pyrochlore spin ice materials, it is antiferromagnetic. So we adopt the notation of AFM here.

${ }^{43}$ R. de Picciotto, M. Reznikov, M. Heiblum, V. Umansky, G. Bunin, and D. Mahalu, "Direct observation of a fractional charge," Nature 389, 162-164 (1997).

44 V. J. Goldman and B. Su, "Resonant tunneling in the quantum hall regime: Measurement of fractional charge," Science 267, 1010-1012 (1995).

45 Xiao-Gang Wen, "Quantum orders and symmetric spin liquids," Phys. Rev. B 65, 165113 (2002).
46 Xiao-Gang Wen, "Quantum order: a quantum entanglement of many particles," Physics Letters A 300, 175 - 181 (2002).

47 Andrew M. Essin and Michael Hermele, "Classifying fractionalization: Symmetry classification of gapped $\digamma_{2}$ spin liquids in two dimensions," Phys. Rev. B 87, 104406 (2013).

48 Andrew M. Essin and Michael Hermele, "Spectroscopic signatures of crystal momentum fractionalization," Phys. Rev. B 90, 121102 (2014).

49 Argha Banerjee, Sergei V. Isakov, Kedar Damle, and Yong Baek Kim, "Unusual Liquid State of Hard-Core Bosons on the Pyrochlore Lattice," Phys. Rev. Lett. 100, 047208 (2008).

50 Jian-Ping Lv, Gang Chen, Youjin Deng, and Zi Yang Meng, "Coulomb Liquid Phases of Bosonic Cluster Mott Insulators on a Pyrochlore Lattice," Phys. Rev. Lett. 115, 037202 (2015).

51 Yasuyuki Kato and Shigeki Onoda, "Numerical Evidence of Quantum Melting of Spin Ice: Quantum-to-Classical Crossover," Phys. Rev. Lett. 115, 077202 (2015).

52 Xiao-Gang Wen, "Quantum orders and symmetric spin liquids," Phys. Rev. B 65, 165113 (2002).

53 Shigeki Onoda and Yoichi Tanaka, "Quantum fluctuations in the effective pseudospin- $\frac{1}{2}$ model for magnetic pyrochlore oxides," Phys. Rev. B 83, 094411 (2011).

54 Yao-Dong Li, Xiaoqun Wang, and Gang Chen, "Hidden multipolar orders of dipole-octupole doublets on a triangular lattice," Phys. Rev. B 94, 201114 (2016). 